\title{
ON THE DIRECT PRODUCT OF $V$-GROUPS
}

\author{
DONALD P. MINASSIAN
}

ABSTRACT. Let $G$ and $H$ be ordered groups such that every full order on a subgroup extends to a full order on the group; then the direct product, $G \times H$, need not have this property. In fact a stronger result holds.

A group is called a $V$-group if every full order on a subgroup may be extended to a full order on the group. Kargapolov [2] showed that an arbitrary torsion-free group $G$ is a $V$-group if and only if $G$ has an abelian normal subgroup $B$ such that (1) the factor group $G / B$ is abelian, and (2) for arbitrary $\beta$ in $B$ and $\alpha$ in $G-B$ there are positive integers $m \neq n$ such that $\alpha^{-1} \beta^{m} \alpha=\beta^{n}{ }^{1}$ In particular, torsion-free abelian groups are $V$-groups. The necessity of Kargapolov's theorem is essentially due to Terehov [6].

Leмma. Let $G$ have the presentation $G=\left\{a, b \mid b a=a b^{p}\right\}$, where $p$ is $a$ fixed prime. Then $G$ is a V-group.

Proof. First of all, $G$ is torsion-free since it admits a full order [1] (direct verification is not difficult). Next, we construct a subgroup $B$ of $G$ satisfying the conditions of Kargapolov's theorem.

By definition of $G, b^{p}=a^{-1} b a$, and so by induction

$$
b^{p k}=a^{-k} b a^{k}, \quad \text { all } k \geqq 0 .
$$

Moreover $\left(a^{k} b a^{-k}\right) p^{k}=a^{k}\left(b^{p^{k}}\right) a^{-k}=b$, so that we may define

$$
b^{p^{-k}} \equiv a^{k} b a^{-k}, \quad \text { all } k \geqq 0 .
$$

It follows that

$$
b^{p^{i}}=a^{-i} b a^{i} \quad \text { for every integer } i \text {. }
$$

Consider the subgroup $B$ of $G$ generated by $\left\{b^{p^{w}}: w\right.$ an integer $\} . B$ consists of all $p$-adic rational powers of $b$, i.e., all $b^{d}$ where $d=$ $\sum_{j} \pm p^{w_{j}}$ for integers $w_{j}$, hence $B$ is torsion-free abelian. Also $B$ is nor-

Received by the editors October 7, 1970.

AMS 1970 subject classifications. Primary 06A55; Secondary 06A55.

Key words and phrases. Partially ordered group, fully ordered group, $V$-group, $V^{*}$. group, $V A N$-group, $V N$-group.

${ }_{1}$ The statement (but not the proof) of this theorem in [2] is incomplete, for it does not mention that $\beta \in B$. Further, the hypotheses cannot be weakened to allow $m, n$ arbitrary integers (see $[4$, p. 226] for a counterexample).

Copyright (c) 1971, American Mathematical Society 
mal in $G$ since $a^{-i} b^{p^{w}} a^{i}=b^{p^{w+i}} \in B$, for all integers $i$ and $w$. Thus given an integer $i \neq 0$ and a $p$-adic rational $d$ there is a $p$-adic rational $d^{\prime} \neq d$ with the same sign as $d$ such that $a^{-i} b^{d} a^{i}=b^{d^{\prime}} \in B$. It follows easily from this that there are positive integers $m \neq n$ with

$$
a^{-i} \beta^{m} a^{i}=\beta^{n} \quad \text { for all } \beta \in B \text {. }
$$

Kargapolov's second condition follows from this once we note that every $g \in G-B$ may be written in the form $g=a^{i} \beta, i \neq 0, \beta \in B$. Q.E.D.

Finally $G / B$ is infinite cyclic, for it is generated by $a B$ and is infinite lest some power of $a$ commute with $b$, violating the definition of $G$.

Theorem. There are $V$-groups $G, H$ with $G \times H$ not a $V$-group.

Proof. Let $G=\left\{a, b \mid b a=a b^{2}\right\}$ and $H=\left\{c, d \mid d c=c d^{3}\right\}$; note that $G$ and $H$ are $V$-groups by the lemma. Were $G \times H$ a $V$-group, it would have an abelian normal subgroup $B$ of the type described in Kargapolov's theorem. Since $(G \times H) / B$ is abelian, $B$ must contain the commutator subgroup $(G \times H)^{\prime}=G^{\prime} \times H^{\prime}$. Setting $i \equiv 1$ in $\left(^{*}\right)$ above shows $b^{2}$ is in $G^{\prime}$ and $d^{2}$ is in $H^{\prime}$. So

$$
\left(b^{2}, d^{2}\right) \in G^{\prime} \times H^{\prime} \subseteq B .
$$

Now

$$
(a, c)^{-1}\left(b^{2}, d^{2}\right)(a, c)=\left(b^{4}, d^{6}\right) .
$$

Thus, if there were positive integers $m \neq n$ such that

$$
(a, c)^{-1}\left(b^{2}, d^{2}\right)^{m}(a, c)=\left(b^{2}, d^{2}\right)^{n},
$$

we would have

$$
\left(b^{4 m}, d^{6 m}\right)=\left(b^{2 n}, d^{2 n}\right)
$$

which is impossible since $G$ and $H$ are torsion-free. Q.E.D.

A group $G$ is called a $V A N$-group if every full order on every abelian normal subgroup extends to a full order on the group. $G$ is called a $V^{*}$-group if every partial order on every subgroup extends to a full order for $G$.

The following corollary to the previous theorem puts it in stronger terms.

Corollary. The direct product of $V^{*}$-groups need not be a VANgroup.

Proof. We claim that a solvable $V A N$-group is a $V^{*}$-group. Assuming this for the moment, the corollary follows, for the group $G \times H$ in the proof of the theorem is solvable and thus, if also $V A N$, is $V^{*}$ and hence $V$. 
It remains to establish the claim. In fact, the classes $V^{*}$ and solvable $V A N$ coincide. A $V^{*}$-group is solvable $V A N$, since $V^{*}$ trivially implies $V A N$ and $V^{*}$, being $V$, implies solvable by Kargapolov's theorem above (note that a $V$-group is torsion-free since it admits a full order). Conversely, a solvable $V A N$-group is $V^{*}$, since a solvable $V N$-group (obvious meaning) is $V^{*}$ (see [3]) and a solvable $V A N$-group is $V N$ [5]. (Terehov [5] actually shows that a solvable $V$-group satisfies conditions (1) and (2) of Kargapolov's theorem, but that proof applies as well to solvable $V A N$-groups; thus a solvable $V A N$-group is $V$ by Kargapolov's theorem, and hence $V N$.)

Acknowledgement. The author wishes to acknowledge the help of the University of Michigan and his thesis advisor, Professor Eugene F. Krause.

\section{REFERENCES}

1. L. Fuchs and E. Sasiada, Note on orderable groups, Ann. Univ. Sci. Budapest. Eötvös Sect. Math. 7 (1964), 13-17. MR 30 \#3925.

2. M. I. Kargapolov, Completely ordered groups, Algebra i Logika Sem. 1 (1962), no. 2, 16-21. (Russian) MR 27 \#2569.

3. A. I. Kokorin, On the theory of completely ordered groups, Ural. Gos. Univ. Mat. Zap. 4 (1963), no. 3, 25-29. (Russian) MR 32 \#1271.

4. D. P. Minassian, Recent developments in the theory of fully ordered groups, Doctoral Thesis, University of Michigan, Ann Arbor, Mich., 1967.

5. A. A. Terehov, Completely orderable groups, Dokl. Akad. Nauk. SSSR 129 (1959), 34-36. (Russian) MR $22 \# 734$.

6. - The structure of locally solvable, completely ordered groups, Algebra i Logika Sem. 1 (1962), no. 2, 10-15. (Russian) MR 27 \#2568.

BUTLER UNIVERSITY, INDIANAPOLIS, INDIANA 46208 